\title{
Consecutive and Extensive Transition of Luminescent Color of an Organic Solid Material by Applying the High Pressure
}

Junjie Guan, ${ }^{\dagger,+}$ Xingyu Tang, ${ }^{\dagger}$ Chunfang Zhang, ${ }^{\S}$ Kuo Li, ${ }^{\dagger}$ Xiaohuan Lin, ${ }^{\dagger}$ Lin

Wang, ${ }^{\dagger, \nabla}$ Hyun Hwi Lee, " Jialiang Xu, ${ }^{*,+, *}$ Haiyan Zheng, ${ }^{*},+$ Ho-kwang Mao ${ }^{\dagger}$

${ }^{\dagger}$ Center for High Pressure Science and Technology Advanced Research (HPSTAR), Beijing 100094, P. R. China

${ }^{\ddagger}$ School of Chemical Engineering and Technology, Tianjin University, Tianjin 300350, P. R. China

${ }^{\S}$ College of Chemistry and Environmental Science, Hebei University, Baoding 071002, Hebei, P. R. China

${ }^{\nabla}$ Center for High Pressure Science (CHiPS), State Key Laboratory of Metastable Materials Science and Technology, Yanshan University, Qinhuangdao 066004, P. R. China

"Pohang Accelerator Laboratory, POSTECH, Pohang 790-784, Republic of Korea

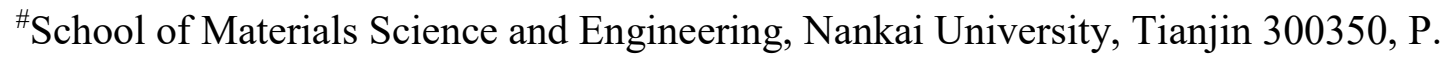
R. China

Emails: jialiang.xu@nankai.edu.cn; zhenghy@hpstar.ac.cn; 


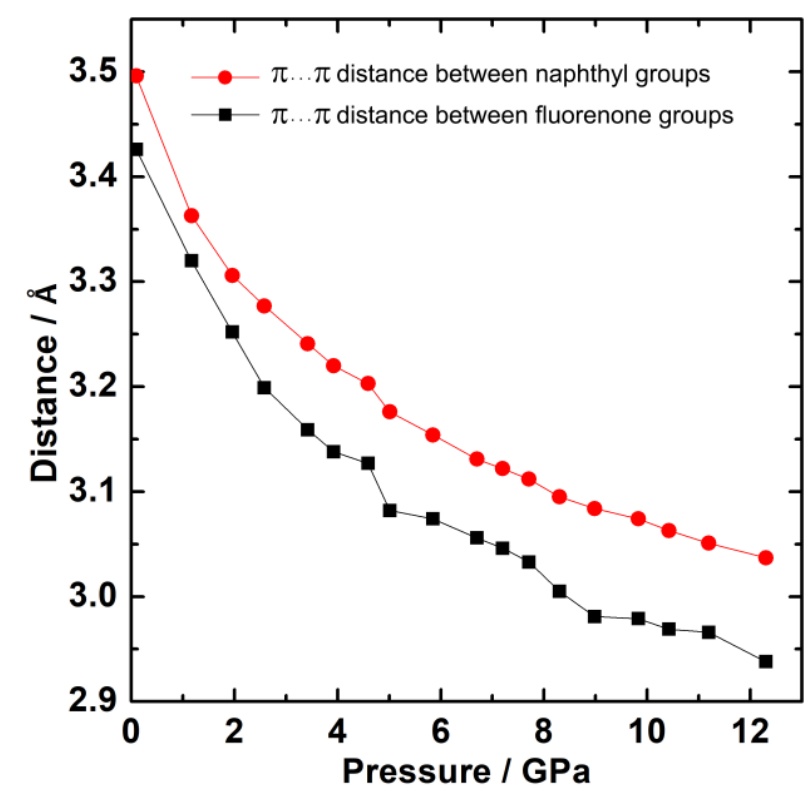

Figure S1. $\pi \cdots \pi$ distances between aromatic rings of 1-DNFO under different pressures.

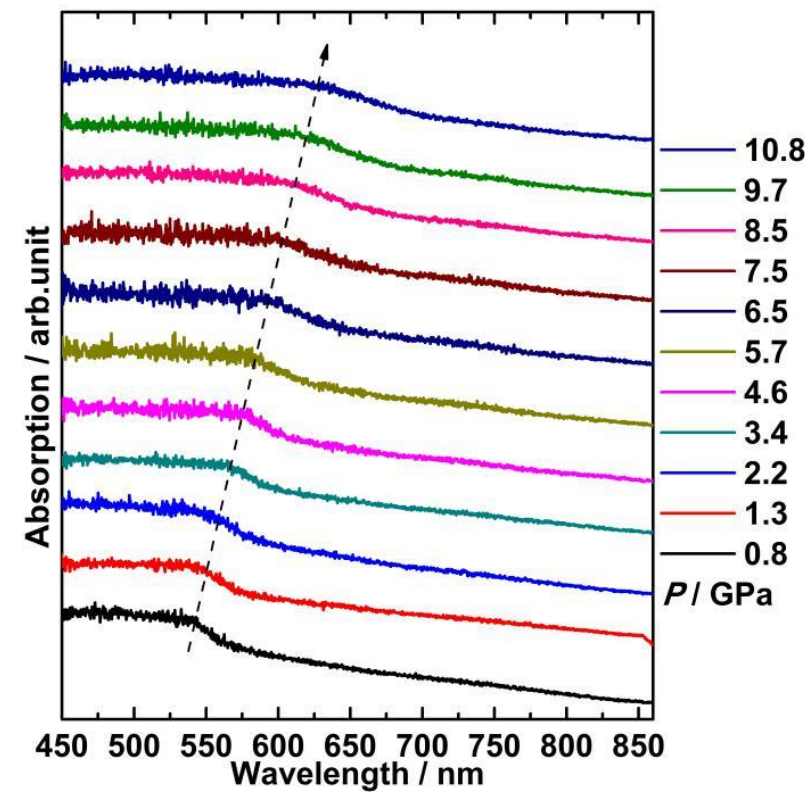

Figure S2. Selected in situ UV-vis absorption spectra of 2-DNFO under different pressures. 


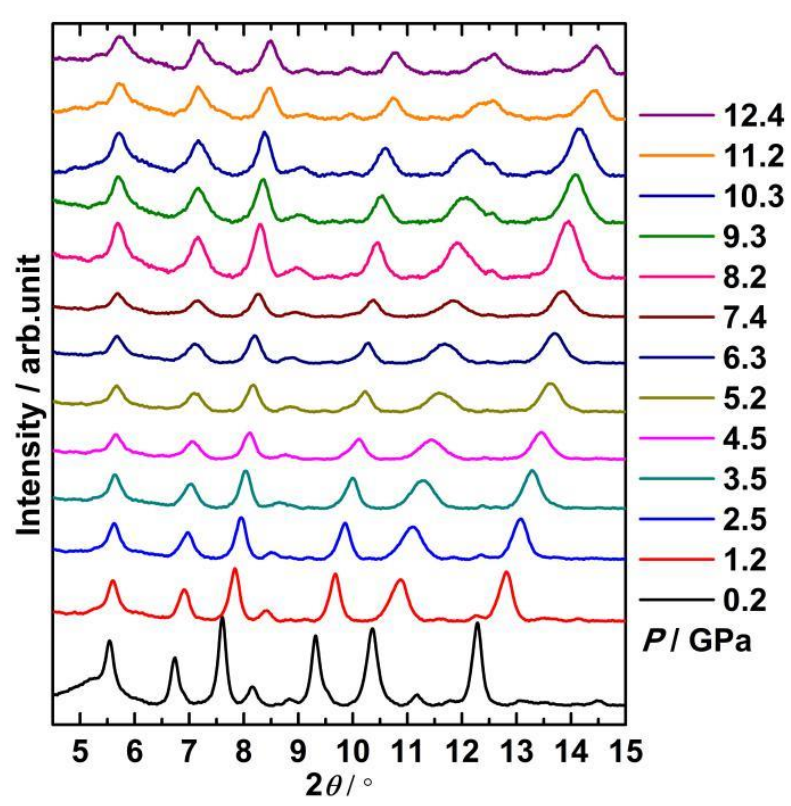

Figure S3. Synchrotron XRD patterns of 2-DNFO with increasing pressure.
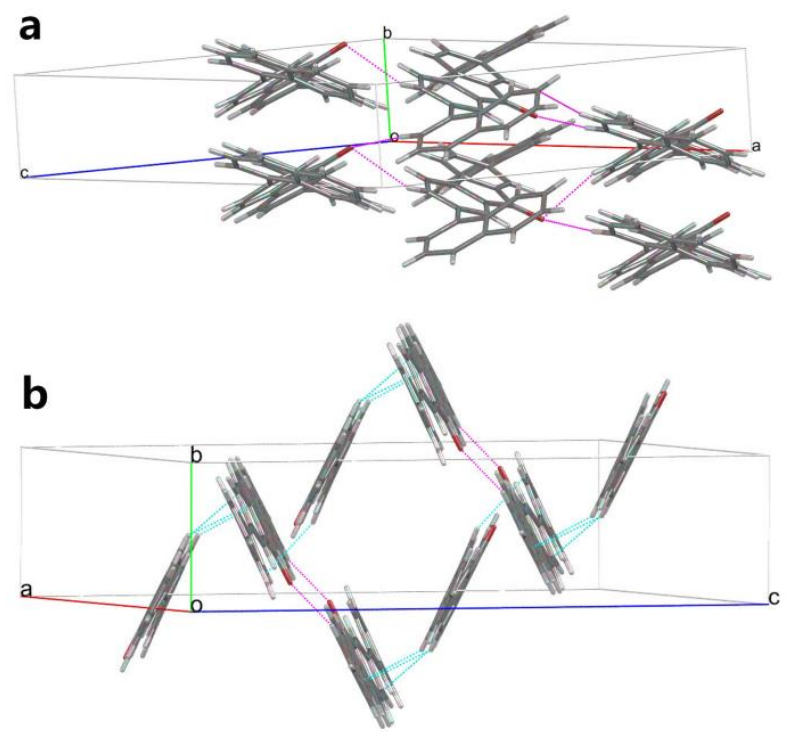

Figure S4. Selected molecular aggregates of (a) 1-DNFO and (b) 2-DNFO in the unit cell (pink dotted lines indicate $\mathrm{C}-\mathrm{H} \cdots \mathrm{O}$ hydrogen bond and blue dotted lines indicate $\mathrm{C}$ $\mathrm{H} \cdots \pi$ interaction). 


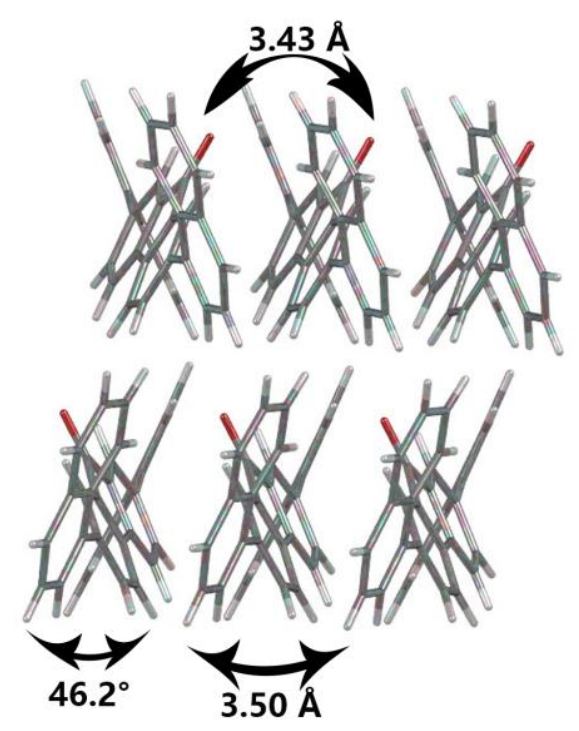

$0.2 \mathrm{GPa}$

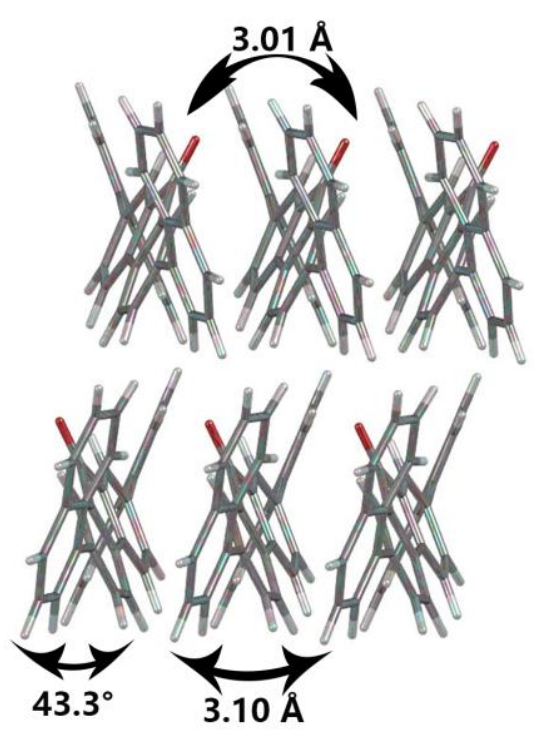

$8.3 \mathrm{GPa}$

Figure S5. The $\pi \cdots \pi$ distance and dihedral angle of selected molecular aggregates of 1DNFO under 0.2 and $8.3 \mathrm{GPa}$, respectively.

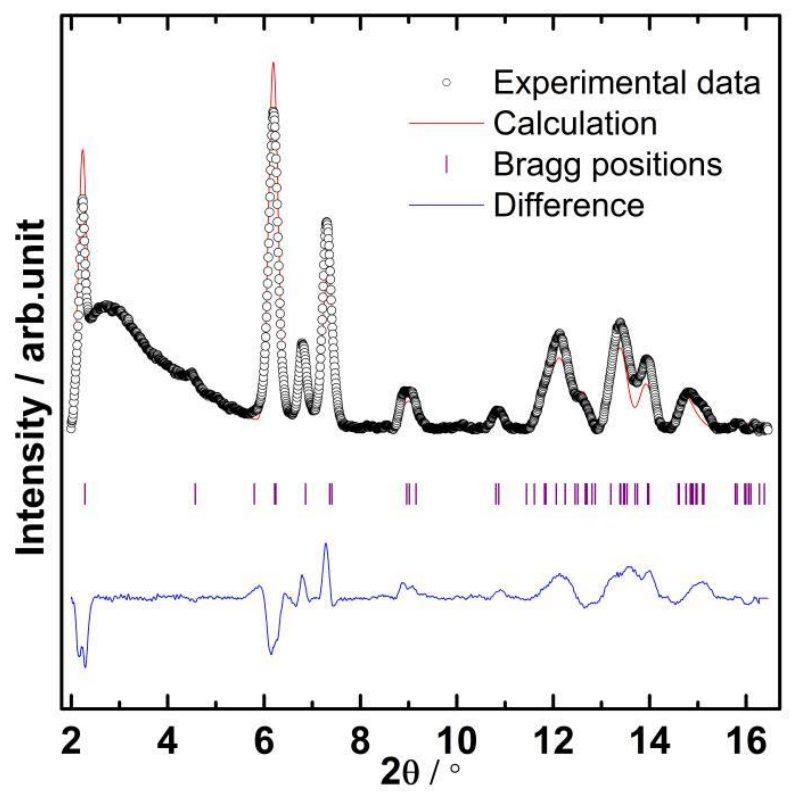

Figure S6. Rietveld refinement result of 1-DNFO at $8.3 \mathrm{GPa}$. 


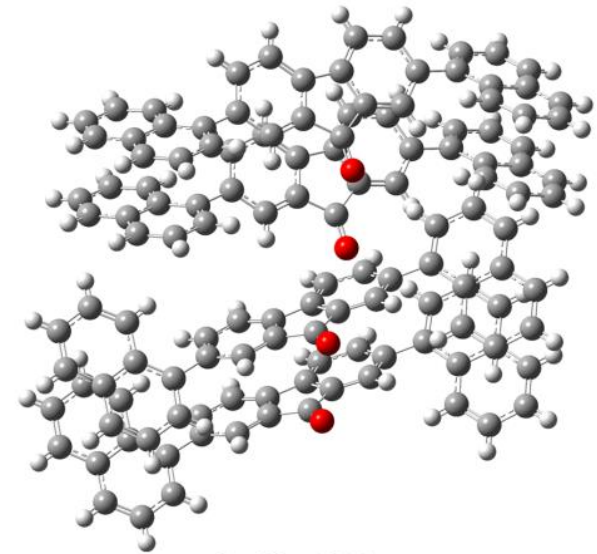

$0.2 \mathrm{GPa}$

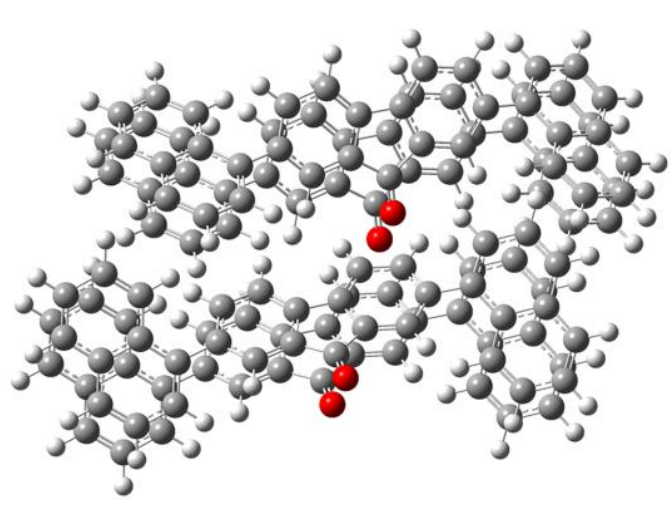

8.3 GPa

Figure S7. Selected models of molecular aggregates of 1-DNFO under 0.2 and $8.3 \mathrm{GPa}$, respectively.

Table S1. The selected molecular orbital energy values of 1-DNFO at 0.2 and $8.3 \mathrm{GPa}$, respectively, obtained from Time-Dependent Density Functional Theory calculation.

\begin{tabular}{ll} 
Molecular orbital & Molecular orbital energy $(\mathrm{eV})$ \\
\hline $0.2 \mathrm{GPa}$ & \\
\hline LUMO & -2.64 \\
HOMO & -5.24 \\
HOMO-1 & -5.34 \\
HOMO-2 & -5.63 \\
HOMO-3 & -5.72 \\
\hline 8.3 GPa & \\
\hline LUMO & -2.92 \\
HOMO & -4.74 \\
HOMO-1 & -5.15 \\
HOMO-2 & -5.17 \\
HOMO-3 & -5.34 \\
\hline
\end{tabular}

